\title{
Spatially autoresonant stimulated Raman scattering in nonuniform plasmas
}

\author{
O. Yaakobi, L. Friedland \\ Racah Institute of Physics, Hebrew University, Jerusalem 91904, Israel \\ R. R. Lindberg, A. E. Charman \\ Department of Physics, U.C. Berkeley, Berkeley, CA 94720 \\ G. Penn, J. S. Wurtele \\ Center for Beam Physics, Lawrence Berkeley National Laboratory, Berkeley, CA 94720
}

LBNL Technical Report, July 2008

Published as Physics of Plasmas 15, 032105 (2008).

\section{Disclaimer}

This document was prepared as an account of work sponsored by the United States Government. While this document is believed to contain correct information, neither the United States Government nor any agency thereof, nor The Regents of the University of California, nor any of their employees, makes any warranty, express or implied, or assumes any legal responsibility for the accuracy, completeness, or usefulness of any information, apparatus, product, or process disclosed, or represents that its use would not infringe privately owned rights. Reference herein to any specific commercial product, process, or service by its trade name, trademark, manufacturer, or otherwise, does not necessarily constitute or imply its endorsement, recommendation, or favoring by the United States Government or any agency thereof, or The Regents of the University of California. The views and opinions of authors expressed herein do not necessarily state or reflect those of the United States Government or any agency thereof, or The Regents of the University of California.

Ernest Orlando Lawrence Berkeley National Laboratory is an equal opportunity employer.

This work was supported by the U.S.-Israel Bi-National Science Foundation, grant number 2004033, by the Director, Office of Science, High Energy Physics, U.S. Department of Energy under Contract No. DE-AC02-05CH11231, by the Advanced Accelerator Concepts initiative of the Division of High Energy Physics, U.S. Department of Energy, grant number DE-FG02-04ER41289, and the National Nuclear Security Administration under the Stewardship Science Academic Alliances Program through the Department of Energy, grant number DE-FG52-07NA28122. 


\title{
Spatially autoresonant stimulated Raman scattering in nonuniform plasmas
}

\author{
O. Yaakobi, ${ }^{1,2}$ L. Friedland, ${ }^{1, *}$ R. R. Lindberg,,${ }^{3, \dagger}$ A. E. Charman, ${ }^{3}$ G. Penn, ${ }^{4}$ and J. S. Wurtele ${ }^{3,4}$ \\ ${ }^{1}$ Racah Institute of Physics, Hebrew University, Jerusalem 91904, Israel \\ ${ }^{2}$ Soreq Research Center, Yavne 81800, Israel \\ ${ }^{3}$ Department of Physics, U.C. Berkeley, Berkeley, CA 94720 \\ ${ }^{4}$ Center for Beam Physics, Lawrence Berkeley National Laboratory, Berkeley, CA 94720
}

(Dated: August 6, 2008)

\begin{abstract}
New solutions to the coupled three-wave equations in a nonuniform plasma medium are presented that include both space and time dependence of the waves. By including the dominant nonlinear frequency shift of the material wave, it is shown that if the driving waves are sufficiently strong (in relation to the medium gradient), a nonlinearly phase-locked solution develops that is characteristic of autoresonance. In this case, the material (electrostatic) wave develops into a front starting at the linear resonance point and moving with the wave group velocity in a manner such that the intensity increases linearly with the propagation distance. The form of the other two (electromagnetic) waves follow naturally from the Manley-Rowe relations.
\end{abstract}

PACS numbers: 52.25.Os, 52.35.-g, 52.35.Fp, 52.35.Hr, 52.35.Mw, 52.38.-r, 52.38.Bv

\section{INTRODUCTION AND MOTIVATION}

Because of their fundamental importance in plasma dynamics (see Ref. [1], Chaps. 6-8), resonant threewave interactions, including stimulated Raman scattering (SRS), stimulated Brillouin scattering (SBS), and other processes, have been widely studied theoretically and numerically. In particular, currently SRS is of great interest as a potentially deleterious reflection mechanism in inertial confinement fusion [2-6], or potentially as a beneficial mechanism for optical pulse compression in plasma-based Raman amplifiers [7, 8].

SRS has been studied mainly in the context of a cw pump or else an eikonal pump wave packet with fixed carrier frequency and wave-number, propagating in a timestationary and spatially homogeneous underdense background plasma. The role of a plasma density gradient in saturating SRS was first studied in Ref. [9]. Recently, Refs. [10] and [11] used chirps in the laser frequency and/or plasma density to suppress deleterous plasma instabilities, while demonstrating that depletion of the pump laser changes the Langmuir wave saturation conditions of Ref. [9] permitting significant energy exchange in the context of transient SRS. These papers neglected any nonlinear effects in the plasma wave itself, assuming that the plasma is sufficiently cold and that depletion happens sufficiently quickly that they may be neglected. In contrast, the autoresonant effect we investigate here arises when the nonlinear frequency shift of the material wave due to thermal and relativistic effects balances that of the plasma gradient. Temporal autoresonance via drive-laser frequency chirping was suggested as a method for excitation of plasma waves [12-14]. Spatially autoresonant three-wave interactions in variable density media were

\footnotetext{
*Electronic address: lazar@vms.huji.ac.il

${ }^{\dagger}$ Electronic address: rlindberg@berkeley.edu
}

introduced about 15 years ago [15], but not in the context of any specific system or application. More recently, autoresonant SBS, including the effects of the damping, ion-trapping and detuning, was considered by Williams et al. [16].

The goal of the present work is to study formation of large amplitude, spatially-autoresonant plasma waves in a non-uniform plasma via SRS. We will discuss the transition to spatial autoresonance during pulsed application of the pump/seed laser waves and the role of the autoresonant threshold phenomenona in this transitional process. Previously, this threshold effect has been studied only in the context of autoresonance in externally-driven dynamical systems and nonlinear wave problems $[17,18]$.

\section{DYNAMICAL MODEL}

\section{A. Three-wave equations with fluid nonlinearity}

Our starting point is a system of envelope equations describing the SRS process in a stationary, onedimensional (along $z$ ), weakly-nonuniform, underdense, thermal plasma with stationary ions (see, e.g., Refs. [4] and [19]):

$$
\begin{aligned}
\mathscr{L}_{a} a & =-\varepsilon \frac{\omega_{g}}{\omega_{a}} b g e^{-i \Psi}, \\
\mathscr{L}_{b} b & =\varepsilon \frac{\omega_{g}}{\omega_{b}} a g^{*} e^{+i \Psi}, \\
\mathscr{L}_{g} g+i \beta|g|^{2} g & =\varepsilon a b^{*} e^{+i \Psi} .
\end{aligned}
$$

Here, the complex envelopes $a, b$, and $g$ describe the pump and seed electromagnetic waves and the plasma Langmuir wave, respectively, and are defined in terms of 
the associated dimensionless rms electric fields via

$$
\begin{aligned}
E_{z} & =\frac{m c}{e} \frac{\omega_{p}(z)}{\sqrt{2}} g e^{i \psi_{g}}+\text { c.c. }, \\
\boldsymbol{E}_{\perp} & =\frac{m c}{e}\left(\frac{\omega_{a}}{\sqrt{2}} a e^{i \psi_{a}}+\frac{\omega_{b}}{\sqrt{2}} b e^{i \psi_{b}}\right) \hat{\boldsymbol{e}}_{\perp}+\text { c.c. },
\end{aligned}
$$

where $c$ is the vacuum speed of light, $m$ is the electron mass, and $e$ the magnitude of its charge; $\hat{\boldsymbol{e}}_{\perp}$ is the common transverse polarization of the laser fields (linear or circular), $\psi_{\ell}=\int^{z} d z^{\prime} k_{\ell}\left(z^{\prime}\right)-\omega_{\ell} t$ for $\ell=a, b, g$, are the eikonal phases of the waves, and $\Psi \equiv \psi_{a}-\psi_{b}-\psi_{g}$. The frequencies $\omega_{\ell}$ of the waves are assumed to be constant and satisfy the three-wave resonance condition $\omega_{a}-\omega_{b}=$ $\omega_{g}$, while the wave vectors are slowly-varying functions of longitudinal position $z$, so as to satisfy the local dispersion relations $\omega_{a, b}^{2}=\omega_{p}^{2}+c^{2} k_{a, b}^{2}$ and $\omega_{g}^{2}=\omega_{p}^{2}+3 v_{\mathrm{th}}^{2} k_{g}^{2}$, where $v_{\text {th }}$ is the electron thermal velocity, assumed uniform, and $\omega_{p}=\omega_{p}(z)$ is the local linear plasma frequency, which is assumed to satisfy $\left|d \omega_{p} / d z\right| \ll \omega_{p}(0) k_{a, b}$. The differential operators

$$
\mathscr{L}_{\ell}=\frac{\partial}{\partial t}+v_{\ell} \frac{\partial}{\partial z}+\frac{1}{2} \frac{\partial v_{\ell}}{\partial z}
$$

are those of slowly-varying linear geometric optics [20], with $v_{a, b}=c^{2} k_{a, b} / \omega_{a, b}$ and $v_{g}=3 v_{\text {th }}^{2} k_{g} / \omega_{g}$ the group velocities of the corresponding waves. The three waves are quadratically coupled via the right-hand sides of Eqs. (1), while the additional term $i \beta|g|^{2}$ represents a nonlinear frequency shift of the plasma wave; the coupling and nonlinearity strengths are given, respectively, by

$$
\varepsilon=\frac{c k_{g} \omega_{p}}{2 \sqrt{2} \omega_{g}}, \quad \beta=\frac{15}{2} \frac{\omega_{g}^{2}}{\omega_{p}^{2}} \frac{v_{\mathrm{th}}^{2} c^{2}}{v_{\mathrm{ph}}^{4}} \omega_{g}-\frac{3}{8} \frac{\omega_{g}^{2}}{\omega_{p}^{2}} \omega_{g},
$$

such that both parameters have dimensions of inverse time. Here $v_{\mathrm{ph}} \equiv \omega_{g} / k_{g}$ is the phase velocity of the plasma wave, and $\beta$ has been calculated in the fluid approximation (see Refs. [21-23]). The first term in $\beta$ arises from the combined effects of the convective nonlinearity $u \partial u / \partial z$ and the thermal spread in the momentum equation, while the second term describes a weaklyrelativistic inertial effect (assuming $|a|^{2},|b|^{2},|g|^{2} \ll 1$ ). We assume that the dimensionless ratio $\eta_{g} \equiv v_{\mathrm{ph}} / v_{\mathrm{th}}$ of the plasma wave phase velocity to electron thermal velocity is sufficiently large (typically, greater than 5 in our examples below) so as to justify neglecting all effects associated with resonant particles, such as Landau damping or other particle-trapping effects, which would effectively contribute additional terms to the nonlinear dispersion $\beta$. Finally, since we assume the resonance condition $\omega_{a}-\omega_{b}=\omega_{g}$ is satisfied, the phase mismatch $\Psi$ in the envelope equations is due entirely to the variation of the wave vectors arising from the plasma nonuniformity, so that $\Psi=\Psi(z)=\int^{z} d z^{\prime}\left[k_{a}\left(z^{\prime}\right)-k_{b}\left(z^{\prime}\right)-k_{g}\left(z^{\prime}\right)\right]$.

In the case of forward scatter, $v_{\mathrm{ph}} \approx c$ and the first term in $\beta$ is much smaller than the second, so that $\beta<0$. In the opposite case of backward scatter, $v_{\mathrm{ph}} \ll c$ so that the first term is typically larger than the second, and $\beta>0$. Here, we focus on the latter scenario of RBS, in which case autoresonant solutions can exist if the plasma density is an increasing function of $z$; for RFS we would require a decreasing plasma gradient.

\section{B. Boundary/initial conditions}

We are interested in analyzing the passage through spatial resonance in the plasma and, consequently, assume that in the vicinity of the resonance at $z=0$, $k_{a}-k_{b}-k_{g} \approx \alpha z$, where $\alpha$ parameterizes the spatial non-uniformity, and is assumed sufficiently small (in a precise sense described below). We solve the system (1) between two fixed plasma boundaries, at $z=z_{\mathrm{L}}<0$ and $z=z_{\mathrm{R}}>0$, and assume that the electromagnetic field $a=a(z, t)$ (pump) is switched on at $z_{\mathrm{L}}$ at time $t=0$, propagates in the positive $z$ direction $\left(v_{a}>0\right)$, remaining at a prescribed constant amplitude at the left boundary $z_{\mathrm{L}}$ for all $t \geq 0$ : i.e., $a\left(z_{\mathrm{L}}, t\right)=\Theta(t) a_{0}$, where $\Theta(t)$ is the Heaviside step function. We suppose the field $b=b(z, t)$ (seed) is also switched on at $t=0$, and either $v_{b}>0$, and $b\left(z_{\mathrm{L}}\right)=\Theta(t) b_{0}$ [for the case of Raman forward scattering (RFS)], or else $v_{b}<0$, and $b\left(z_{\mathrm{R}}, t\right)=\Theta(t) b_{0}$ [for the case of Raman backward scattering (RBS)]. Finally, the plasma wave is initially zero, i.e., $g(z, t=0)=0$. We seek the solution of this initial/boundary problem in the space-time domain $z_{\mathrm{L}} \leq z \leq z_{\mathrm{R}}, t \geq 0$.

\section{Rescaling}

At this stage, we rewrite the coupled equations (1) in a more convenient dimensionless form. We introduce the dimensionless longitudinal coordinate $\xi \equiv \sqrt{|\alpha|} z$ scaled to the plasma gradient, and the dimensionless time $\tau \equiv$ $v_{g}(0) \sqrt{|\alpha|} t$. Furthermore, we define the scaled action amplitudes of the waves,

$$
\begin{aligned}
A(\xi, \tau) & =\frac{\sqrt{v_{a}}}{\sqrt{v_{a}\left(z_{\mathrm{L}}\right)}} \frac{a(z, t)}{a_{0}}, \\
B(\xi, \tau) & =\frac{\sqrt{\left|v_{b}\right|}}{\sqrt{v_{a}\left(z_{\mathrm{L}}\right)}} \frac{\sqrt{\omega_{b}}}{\sqrt{\omega_{a}}} \frac{b(z, t)}{a_{0}}, \\
G(\xi, \tau) & =\frac{\sqrt{v_{g}}}{\sqrt{v_{a}\left(z_{\mathrm{L}}\right)}} \frac{\sqrt{\omega_{g}}}{\sqrt{\omega_{a}}} \frac{g(z, t)}{a_{0}} e^{i \xi^{2} / 2},
\end{aligned}
$$

which simplify both the advection operators $\mathscr{L}_{\ell}$ and the coupling parameters. In these dependent and independent variables, the scaled governing equations become

$$
\begin{aligned}
\frac{v_{g}}{v_{a}} \frac{\partial A}{\partial \tau}+\frac{\partial A}{\partial \xi} & =-\tilde{\varepsilon} B G, \\
\frac{v_{g}}{\left|v_{b}\right|} \frac{\partial B}{\partial \tau}+\sigma \frac{\partial B}{\partial \xi} & =\tilde{\varepsilon} A G^{*}, \\
\frac{\partial G}{\partial \tau}+\frac{\partial G}{\partial \xi}-i \sigma\left(\tilde{\beta}|G|^{2}-\xi\right) G & =\tilde{\varepsilon} A B^{*},
\end{aligned}
$$


where $\sigma=\operatorname{sgn}\left(v_{b}\right)$, and $\tilde{\varepsilon}$ and $\tilde{\beta}$ are dimensionless parameters describing respectively the wave coupling and plasma nonlinearity:

$$
\tilde{\varepsilon}=\frac{a_{0} \sqrt{\omega_{g}}}{\sqrt{|\alpha| \omega_{b} v_{g} v_{b}}} \varepsilon, \quad \tilde{\beta}=\frac{a_{0}^{2} \omega_{a} v_{a}}{\sqrt{|\alpha| \omega_{g} v_{g}^{2}}}|\beta| .
$$

As discussed previously, the sign of $\beta$ (and, hence, $\alpha$ ) depends on whether one considers forward or backward scattering, with $\operatorname{sgn}(\beta)=-\sigma$. Thus, note that Eq. (4c) incorporates the signs of $\alpha$ (the plasma gradient) and $\beta$ (the nonlinearity) using $\sigma$. In our new notation, the boundary conditions become $A\left(\xi_{\mathrm{L}}, \tau\right)=\Theta(\tau)$ and either $B\left(\xi_{\mathrm{L}}, \tau\right)=B_{0} \Theta(\tau)$ (for RFS) or $B\left(\xi_{\mathrm{R}}, \tau\right)=B_{0} \Theta(\tau)$ (for RBS). For simplicity we will assume that all parameters in Eqs. (4a)-(4c) are constant in the following discussion.

\section{AUTORESONANT SOLUTIONS}

\section{A. Time-stationary case}

To introduce our subsequent analysis and make connection with previous work, we first consider the stationary (i.e., time-independent) solution of Eqs. (4). In this case, we have the simplified set of equations

$$
\begin{aligned}
\frac{\partial A}{\partial \xi} & =-\tilde{\varepsilon} B G, \\
\sigma \frac{\partial B}{\partial \xi} & =\tilde{\varepsilon} A G^{*}, \\
\frac{\partial G}{\partial \xi}-i \sigma\left(\tilde{\beta}|G|^{2}-\xi\right) G & =\tilde{\varepsilon} A B^{*},
\end{aligned}
$$

where $A, B$, and $G$ depend only on $\xi$. This set of equations was studied in Ref. [15] for RFS $(\sigma=+1)$, where it was demonstrated, under certain criteria, to yield spatially autoresonant solutions. These solutions are typified by two effects: the nonlinearity of the plasma wave effectively cancels the plasma gradient in Eq. (5c), so that $|G|^{2} \approx \xi / \tilde{\beta}$, while the relative phase of the three coupled modes remains nearly invariant apart from some small oscillations,

$$
\Phi \equiv \arg (A)-\arg (B)-\arg (G) \approx \text { constant } .
$$

Autoresonant nonlinear phase-locking, for which the relative phase between waves is given by expressions analogous to Eq. (6), was first observed in nonneutral plasma experiments [24]. These experiments (and the corresponding theory) demonstrated that as the system passes through resonance, the persistence of nonlinear phase-locking (autoresonance) requires that the normalized driving amplitude $\mu$ be sufficiently large at the linear resonance to overcome a given nonlinearity, such that $\mu>\mu_{\mathrm{cr}} \equiv 0.41 \tilde{\beta}^{-1 / 2}$, in order for phase-locking to occur. In our three-wave scenario with a nonlinear plasma wave, we see from Eq. (5c) that this threshold is characterized
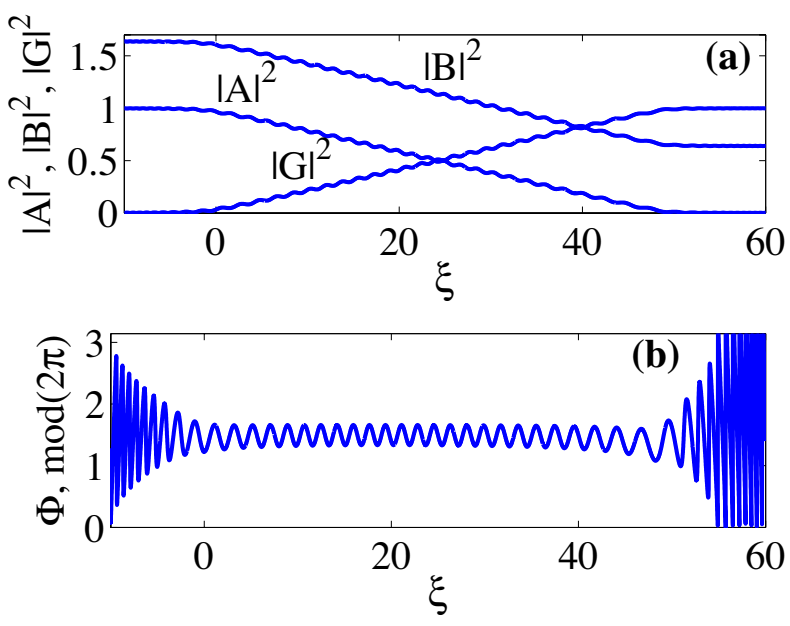

FIG. 1: (Color online) Spatially autoresonant evolution: (a) Normalized wave intensities $|A|^{2},|B|^{2}$ and $|G|^{2}$ vs. scaled longitudinal coordinate $\xi$. (b) Phase mismatch $\Phi$ vs. $\xi$.

by the scaled ponderomotive drive $\mu \equiv \tilde{\varepsilon}|A B|$. For the case of RFS, one can use the value of $|A B|$ given at the boundary $\xi_{\mathrm{L}}$ to estimate this threshold.

The autoresonant time-independent solutions of Eqs. (5) for the RBS $(\sigma=-1)$ case are similar to those in the RFS geometry if the standard autoresonant threshold condition is satisfied at the linear resonance, i.e., if $\mu>\mu_{\mathrm{cr}}$ at $\xi_{\mathrm{L}}$. However, there exists a complication since the RBS scenario involves a mixed boundary-value problem, with the initial $A_{0}=1$ given at $\xi_{\mathrm{L}}$ and $B_{0}$ prescribed at $\xi_{\mathrm{R}}$. This may result in a multiplicity of possible time independent solutions for given $A_{0}$ and $B_{0}$ as discussed below. Nevertheless, if $B_{0}$ is sufficiently large there exists a single autoresonant solution in the RBS problem. We present such a RBS solution for the dimensionless wave intensities in Fig. 1(a), for which we solve the stationary Eqs. (5) assuming two near-10 $\mu \mathrm{m}$ pump and seed lasers of amplitudes $a_{0}=0.00316$ and $b_{0}=0.80 a_{0}$ at the appropriate boundaries (corresponding to laser intensities of $1.37 \times 10^{11}$ and $\left.0.877 \times 10^{11} \mathrm{~W} / \mathrm{cm}^{2}\right)$ and $G_{0}=0$ at $\xi_{\mathrm{L}}$. We assume a $100 \mathrm{eV}$ plasma with a central density of $n_{0}=5 \times 10^{17} \mathrm{~cm}^{-3}$ and plasma density spatial variation scale-length $L=1 \mathrm{~cm}$ (where we define $\left.L^{-1} \equiv n_{0}^{-1} d n_{0} / d z\right)$. In this case $\alpha \approx \omega_{p}^{2}\left(6 k_{g} L v_{\mathrm{th}}^{2}\right)^{-1}=$ $6 \times 10^{4} \mathrm{~cm}^{-2}, \eta_{g}=5.35, \tilde{\beta}=48.2$, and $\tilde{\varepsilon}=0.25$, while $A\left(\xi_{\mathrm{L}}\right)=1, B\left(\xi_{\mathrm{R}}\right)=0.8$, with $\xi_{\mathrm{L}}=-10$ and $\xi_{\mathrm{R}}=+60$, corresponding to $z_{\mathrm{R}}-z_{\mathrm{L}}=0.26 \mathrm{~cm}$.

In Fig. 1, we observe that beyond the linear resonance at $\xi=0$, the plasma wave intensity grows in an approximately linear fashion, $|G|^{2} \approx \xi / \tilde{\beta}$, so that the nonlinear shift in wavevector balances the linear dispersion due to the plasma nonuniformity in Eq. (5c). At the same time, the three waves are continuously phase-locked, i.e., $\Phi \approx \pi / 2$, as shown in Fig. 1(b). Furthermore $|A|^{2}$ and $|B|^{2}$ are also approximately linear functions of $\xi$ due to 


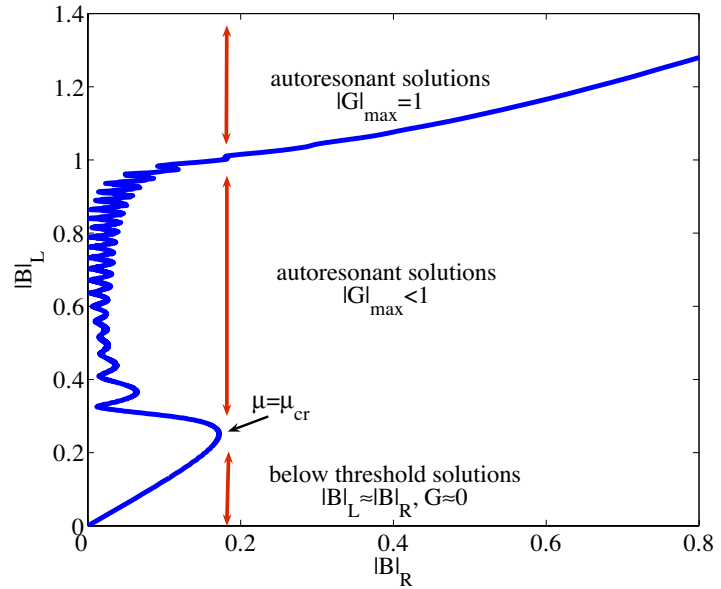

FIG. 2: (Color online) The seed wave amplitude $|B|_{\mathrm{L}}$ at $\xi_{\mathrm{L}}$ vs. the seed amplitude $|B|_{\mathrm{R}}$, in the time-independent mixed boundary-value problem. Note the multiplicity of solutions for $|B|_{\mathrm{R}} \lesssim 0.175$.

the stationary Manley-Rowe conditions,

$$
\begin{aligned}
|A|^{2}+|G|^{2} & =\text { constant } \\
|B|^{2}-\sigma|G|^{2} & =\text { constant. }
\end{aligned}
$$

On top of the average linear growth of the wave intensities appear small oscillations characteristic of autoresonance (discussed further below), whose slowlyvarying frequency scales as $\tilde{\varepsilon}^{1 / 2}$. After essentially all the pump action has been transferred to the plasma wave, so that $|A|^{2} \approx 0$ and $|G|^{2} \approx 1$, autoresonance is lost. This happens near the point $\xi \approx \tilde{\beta}$, and in our example corresponds to a longitudinal electric field of the Langmuir wave given by $E_{z} \approx 0.54 E_{0}$, where $E_{0} \equiv\left[v_{\mathrm{ph}} / v_{a}(0)\right]\left[m c \omega_{p}(0) / e\right]$ is the usual cold, nonrelativistic wavebreaking limit near resonance.

As alluded to earlier, the autoresonant scenario depicted in Fig. 1 and discussed above typifies the timeindependent RBS solutions of Eqs. (5) when $B_{0}$ is sufficiently large. For smaller $B_{0}$ one encounters a multiplicity of time-independent solutions due to the mixed boundary-value problem in RBS case, with the initial $A_{0}$ given at $\xi_{\mathrm{L}}$ and $B_{0}$ prescribed at $\xi_{\mathrm{R}}$. To illustrate this, we solve the (inverse) "initial" value problem (in $\xi$ ), assuming that we are given $A\left(\xi_{\mathrm{L}}\right)$ and $B\left(\xi_{\mathrm{L}}\right)$. We plot the resulting dependence between the seed amplitudes $|B|_{\mathrm{L}} \equiv\left|B\left(\xi_{\mathrm{L}}\right)\right|$ and $|B|_{\mathrm{R}} \equiv\left|B\left(\xi_{\mathrm{R}}\right)\right|$ on the two boundaries in Fig. 2. One can see in the figure that only if $|B|_{\mathrm{L}}>1$ and $|B|_{\mathrm{R}} \gtrsim 0.175$ in our example does one obtain a single, autoresonant solution. In this case, at $\xi_{\mathrm{L}}, \mu=\tilde{\varepsilon}|A B|_{\mathrm{L}}>1.72 \tilde{\beta}^{-1 / 2}$, a more stringent condition than that for the RFS case (where $\mu$ must exceed $\mu_{\mathrm{cr}}=0.41 \tilde{\beta}^{-1 / 2}$ to access autoresonance). For smaller values of $|B|_{\mathrm{R}}$, there exists a multiplicity of solutions naturally separated into different regimes as indicated by double arrows in Fig. 2. For $\mu<\mu_{\mathrm{cr}}$, no significant excitation of $G$ is observed, so $|B|_{\mathrm{L}} \approx|B|_{\mathrm{R}}$. For larger $\mu$, when $|B|_{\mathrm{L}} \in(0.23,1)$ in the figure, spatially autoresonant plasma wave solutions exist, but do not reach the maximum $|G|=1$ at $\xi=\tilde{\beta}^{1 / 2}$ as in Fig. 1, because the phase-locking in the "inverse" problem is lost, as the seed is nearly depleted at some $\xi<\tilde{\beta}^{1 / 2}$. Only for $|B|_{\mathrm{L}}>1$ does one obtain a spatially autoresonant plasma wave reaching its maximum possible amplitude of $|G|=1$ at $\xi=\tilde{\beta}^{1 / 2}$ as in Fig. 1. However, because of the multiplicity of time-independent solutions of the mixed boundary value problem in the RBS case, the stationary problem (5) is physically indeterminate, and we must consider the full, time-dependent problem to determine which asymptotic state will be achieved.

\section{B. RBS in the full, one-dimensional case}

We now numerically solve the fully space-time dependent, initial/boundary three-wave problem (4) for the case of RBS, and find naturally-arising, quasi-stationary solutions that have many of the same essential features as the autoresonant solutions in the time-independent case studied above. We show the resulting wave intensities and relative phase $\Phi$ as functions of $\xi$ for three different scaled times $\tau=10,20,30$ in Fig. 3, using the same parameters and boundary conditions as in Fig. 1, but with the pump and seed waves switched on suddenly at $\tau=0$. Where it is excited, the plasma wave $|G|^{2}$ is again a nearly linear function of $\xi$, except in this case it has a steep front moving with the group velocity. Assuming an underdense plasma at moderate temperatures (such that the group velocity of the EM fields far exceeds that of the plasma wave), the location of the front is approximately $\xi_{\mathrm{f}}=\xi_{\mathrm{f}}(\tau) \approx \tau$, and therefore, prior to the depletion of the pump at $\xi=\tilde{\beta}$, the plasma wave can be approximated by

$$
|G(\xi, \tau)|^{2} \approx \begin{cases}\xi / \tilde{\beta} & \text { if } 0<\xi<\xi_{\mathrm{f}}(\tau) \\ 0 & \text { otherwise }\end{cases}
$$

At the same time, because of the near stationarity of the solution behind the front, the amplitudes $|A|^{2}$ and $|B|^{2}$ for the pump and seed waves approximately satisfy the algebraic Manley-Rowe conditions of the time-independent problem (7), and thus also evolve approximately linearly in $\xi$.

\section{Prescribed ponderomotive drive model}

The formation of autoresonant plasma waves in nonuniform plasmas as illustrated above can be understood more simply by considering the case of a prescribed ponderomotive drive governed by [compare to Eq. (4c)]

$$
\frac{\partial G}{\partial \tau}+\frac{\partial G}{\partial \xi}+i\left(\tilde{\beta}|G|^{2}-\xi\right) G=F(\xi, \tau),
$$




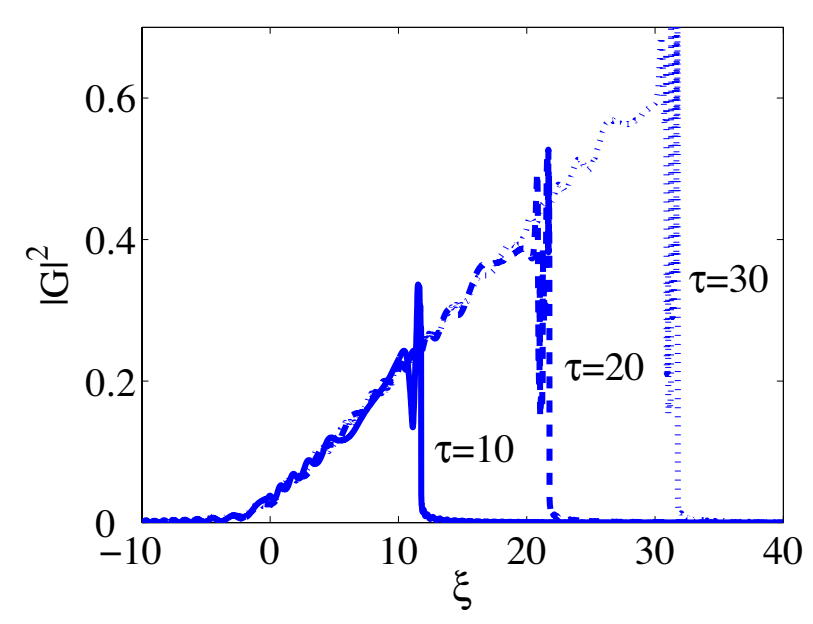

FIG. 3: (Color online) Solutions of the full three-wave system as a function of $\xi$ at different times: $\tau=10,20,30$.

where $F=F(\xi, \tau)$ is a given slow function of $\xi$ and $\tau$, corresponding to the large pump and large seed limit. This partial differential equation can be solved along the characteristics defined via $d \xi / d s=1, d \tau / d s=1$, with $s$ a real parameter chosen such that $s=0$ at $\xi=0$. Thus, the characteristics comprise a set of straight lines defined by $\xi=s, \tau=s-s_{*}$ in the $(\xi, \tau)$-plane between the two boundaries $\xi_{\mathrm{L}}$ and $\xi_{\mathrm{R}}$, where we have labeled each characteristic by its $\xi$-intercept $s_{*}$. Then, Eq. (9) becomes the ordinary differential equation

$$
\frac{d}{d s} Y+i\left(|Y|^{2}-s\right) Y=\mu^{\prime}(s)
$$

along these characteristics, where $Y=Y(s) \equiv \tilde{\beta}^{1 / 2} G$ and $\mu^{\prime} \equiv \tilde{\beta}^{1 / 2} F$. To obtain $Y(\xi, \tau)$, one must solve Eq. (10) subject to the "initial" condition $\left.Y\right|_{s=\xi_{\mathrm{L}}}=0$ for each $s_{*} \in\left[\xi_{\mathrm{L}}, \xi_{\mathrm{R}}\right]$. For constant $\mu^{\prime}$, this driven, single parameter nonlinear Schrödinger equation is the archetype of all weakly nonlinear dynamical autoresonance problems and has been studied extensively in the past (see, for example, Ref. [17]). One of the important results of these studies is a pronounced threshold phenomenon: starting with $Y=0$ sufficiently far from the linear resonance, i.e., at a large negative value of $s$, there exists a threshold value of $\mu_{\mathrm{cr}}^{\prime} \equiv 0.41$, such that if $\left|\mu^{\prime}\right|>\mu_{\mathrm{cr}}^{\prime}$, the driven solution is characterized by a phase-locked $[\arg (Y) \approx$ const], continuously growing solution with $|Y|^{2} \approx s$ beyond the linear resonance. In contrast, below the threshold, the solution of Eq. (10) dephases from the drive near the linear resonance and $|Y|$ saturates at a relatively small value. For $\left|\mu^{\prime}\right|>\mu_{\mathrm{cr}}^{\prime}$, a smooth, averaged autoresonant growth of $|Y|$ is accompanied by characteristic oscillations around this average. The frequency of these oscillations scales as $\nu \approx 2 \sqrt{\mu|Y|}$. The phase mismatch $\Phi$ also oscillates with this frequency.

The RBS case under consideration differs from the canonical example described above by the dependence

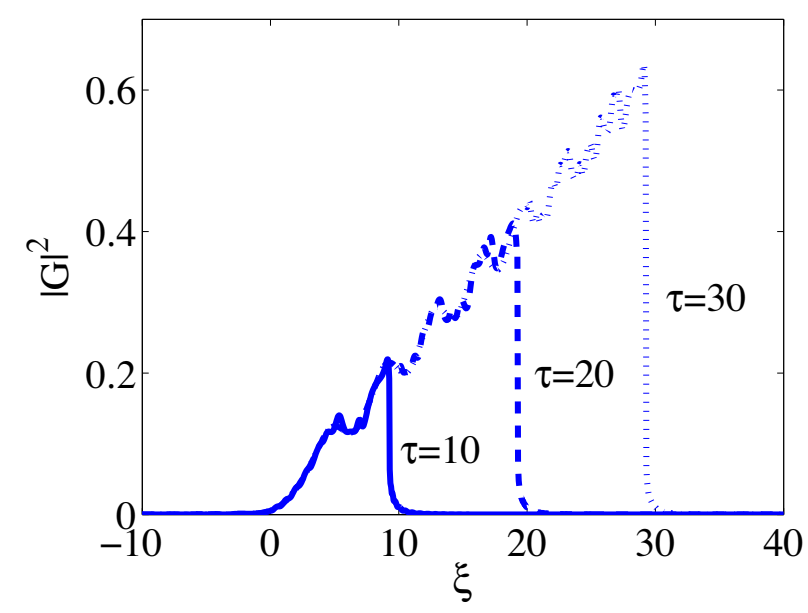

FIG. 4: The normalized plasma wave intensity vs. $\xi$ at different times, $\tau=10,20$, 30, with a prescribed drive $\mu^{\prime}$ that rises suddenly from zero to $\mu_{0}$ at $\tau=0$.

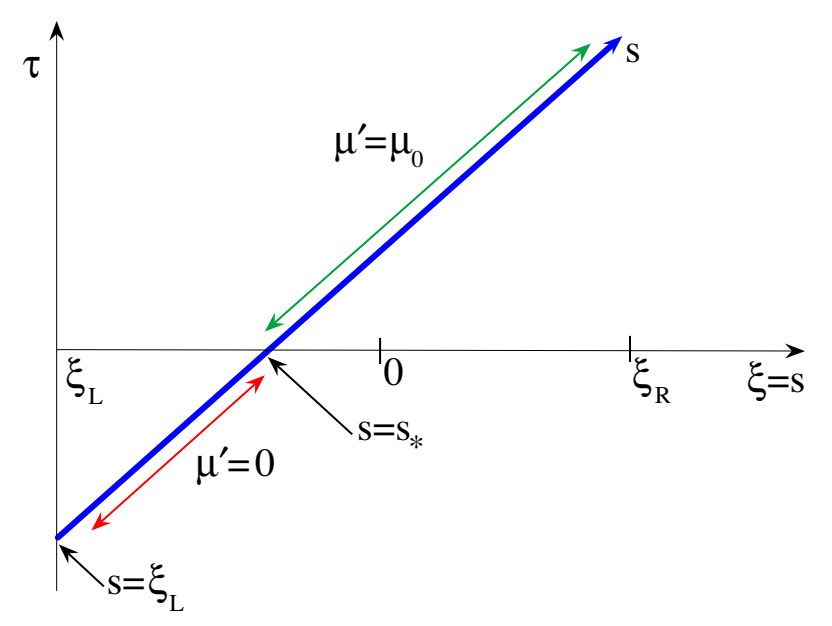

FIG. 5: (Color online) A typical characteristic of the externally forced plasma wave equation in the $\xi-\tau$ plane for which phase-locking can be achieved. The drive $\mu^{\prime}$ switches on at the point $s=s_{*}<0$, before passage through resonance at $\xi=0$.

of the scaled drive $\mu^{\prime}$ on $\xi$ and $\tau$, i.e., on $s$. Figure 4 shows an example of the evolution of $G(\xi, \tau)$ obtained by integrating Eq. (10) for a $\mu^{\prime}$ that is constant in $\xi$, but a step function in time: $\mu^{\prime}=\mu_{0} \Theta(\tau)$. We used $\mu_{0}=0.46$ and, as in Fig. 3, $\xi_{\mathrm{L}}=-10$. Comparing the solution of $|G|^{2}$ in Fig. 4 to that obtained solving the full three-wave problem in Fig. 3, we observe a remarkable similarity between the two solutions, which we explain as follows.

Consider the characteristic determined by a given intercept $s_{*}$ as shown in Fig. 5. The plasma wave is initially zero when the drive is suddenly turned on at $\tau=0$ (or equivalently, $s=s_{*}$ ), so that $Y=0$ everywhere along the characteristic where the parameter $s \leq s_{*}$. As $s \rightarrow \infty$, Eq. (10) has two asymptotic solutions: the saturated, 
constant amplitude solution $Y=Y_{0} e^{i s^{2} / 2}$, and the growing (autoresonant) solution $|Y|^{2}=s$. Thus, the problem reduces to determining which asymptotic solution is approached, subject to the initial condition $Y\left(s_{*}\right)=0$ for each $s_{*}$, i.e., at different locations relative to the linear resonance point $s=0$. For each value of $s_{*}$, the scaled drive $\mu^{\prime}$ determines the asymptotic solution. For those characteristics with $s_{*}<0$, the drive appears before the linear resonance is passed, so that Eq. (10) behaves as in the previously-studied case of a driven, nonlinear oscillator, yielding autoresonant solutions if $\mu_{0}>\mu_{\mathrm{cr}}^{\prime}$. For $s_{*}>0$, in contrast, the drive is turned on only after the linear resonance has been passed, and autoresonance is impossible. This behavior explains the triangular front seen in Fig. 4: the front is located at $\xi \approx \tau$, for which $s_{*} \approx 0$; ahead of the front, $s_{*}>0$, autoresonance cannot occur, and the plasma wave amplitude is small. Behind the front, $s_{*}<0$, the autoresonant solution exists, with $|G|^{2} \approx \xi / \tilde{\beta}$. This in turn explains the features seen in the solutions of the full three-wave problem plotted in Fig. 3. For those plasma-wave characteristics that pass the resonance point before the drive is turned on, the amplitude remains small. On the other hand, if the characteristics pass the resonance point when the ponderomotive drive strength is sufficiently large, so that $\tilde{\varepsilon} \tilde{\beta}^{1 / 2}|A B|>\bar{\mu}$, the relative phase $\Phi \approx$ const, the plasma-wave nonlinearity cancels the change in the Langmuir dispersion, and $|G|^{2} \approx \xi / \tilde{\beta}$. This is the process by which the fully time-dependent problem chooses from the multiplicity of steady-state solutions shown in Fig. 2, and indicates that autoresonance prevails for those characteristics that pass the linear resonance after the drive has turned on, provided that the drive is sufficiently strong such that $\mu>\mu_{\text {cr }}$, as in the RFS case.

\section{CONCLUSIONS}

To summarize, provided $\tilde{\varepsilon} \tilde{\beta}^{1 / 2}|B|_{\mathrm{R}}>\mu_{\text {cr }} \approx 0.41$, the excited plasma wave in a nonuniform plasma has a simple form represented by Eq. (8), with a maximum squared amplitude growing linearly in $\xi$ behind a front that moves with the group velocity of the wave. In the case of Raman backscatter, this condition can be approximately written in terms of physical variables (namely, the plasma gradient scale length $L$, temperature $T_{e}$, and density $n_{e}$, along with the two laser intensities $I_{0}, I_{1}$, and their central wavelength $\lambda$ ) in the following manner

$$
\begin{aligned}
& \frac{c}{v_{\mathrm{th}}}\left(\frac{\omega_{g} L}{v_{\mathrm{ph}}}\right)^{1 / 4}\left(\frac{\omega_{g} L}{v_{\mathrm{th}}}\right)^{1 / 2}\left(\frac{\beta}{\omega_{g}}\right)^{1 / 2} \frac{a_{0} b_{0}}{6^{3 / 4} \mu_{\mathrm{cr}}} \\
& \approx \frac{(L[\mathrm{~cm}])^{3 / 4}\left(I_{0} I_{1}\left[10^{10} \mathrm{~W} / \mathrm{cm}^{2}\right]\right)^{1 / 2}}{\left(\lambda[\mu \mathrm{m}] T_{e}[\mathrm{eV}]\right)^{1 / 4}\left(n_{e}\left[10^{18} \mathrm{~cm}^{-3}\right]\right)^{3 / 4}}>1 .
\end{aligned}
$$

Since the plasma wave behind the front is nearly stationary, the algebraic Manley-Rowe conditions (7) appopriate for a steady-state effectively determine the in- tensities of the autoresonant pump and seed waves. Provided that the above threshold condition is satisfied, the nonlinear phase-locking is insensitive to the spatiotemporal form and/or strength of the ponderomotive drive, so that $\tilde{\beta}|G|^{2}-\xi \approx 0$ throughout the autoresonant plasma region.

In physically realizable systems, there will be additional laser structure including amplitude variations due to the perpendicular envelope and speckles, along with laser diffraction and three-dimensional plasma inhomogeneities. Because of the sharp threshold criterion, autoresonant behavior will arise only where the laser amplitude is sufficiently strong and the plasma gradient sufficiently mild such that the condition (11) is satisfied locally. Thus, realistic variations may lead to interesting three-dimensional spatiotemporal dynamics in which there may be several localized regions of autoresonant behavior. Finally, large amplitude plasma waves may be subject to the modulational instability when $\beta\left(d^{2} \omega_{g} / d k^{2}\right)<0$. In the RBS case discussed above, the nonlinear frequency shift is positive, so that the plasma wave is necessarily modulationally stable. In contrast, $\beta<0$ in the RFS geometry, so that in this case the plasma wave is unstable to sufficiently long wavelength perturbations. Thus, except for a small window of parameters in which stability is provided by the autoresonant driving [25], RFS autoresonance would require laser pulses that are shorter than the characteristic time-scale of the modulational instability. Studying such additional effects may provide interesting directions for future research.

\section{ACKNOWLEDGMENTS}

This work was supported by the U.S.-Israel BiNational Science Foundation, grant number 2004033, by the Director, Office of Science, High Energy Physics, U.S. Department of Energy under Contract No. DEAC02-05CH11231, by the Advanced Accelerator Concepts initiative of the Division of High Energy Physics, U.S. Department of Energy, grant number DE-FG0204ER41289, and the National Nuclear Security Administration under the Stewardship Science Academic Alliances Program through the Department of Energy, grant number DE-FG52-07NA28122.

\section{References}

[1] W. L. Kruer, The Physics of Laser Plasma Interactions (Westview Press, Cambridge, MA, 2001).

[2] J. Lindl, Phys. Plasmas 2, 3933 (1995).

[3] J. F. Drake, P. K. Kaw, Y. C. Yee, G. Schmidt, C. S. Liu, and M. N. Rosenbluth, Phys. Fluids 17, 778 (1974).

[4] D. W. Forslund, J. M. Kindel, and E. L. Lindman, Phys. Fluids 18, 1002 (1975). 
[5] D. W. Phillion, D. L. Banner, E. M. Campbell, R. E. Turner, and K. G. Estabrook, Phys. Fluids 25, 1434 (1982).

[6] J. C. Fernández, J. A. Cobble, D. S. Montgomery, M. D. Wilke, and B. B. Afeyan, Phys. Plasmas 7, 3743 (2000).

[7] G. Shvets, N. J. Fisch, A. Pukhov, and J. Meyer-ter Vehn, Phys. Rev. Lett. 81, 4879 (1998).

[8] V. M. Malkin, G. Shvets, and N. J. Fisch, Phys. Rev. Lett. 82, 4448 (1999).

[9] M. N. Rosenbluth, R. B. White, and C. S. Liu, Phys. Rev. Lett. 31, 1190 (1973).

[10] V. M. Malkin, G. Shvets, and N. J. Fisch, Phys. Rev. Lett. 84, 1208 (2000).

[11] V. M. Malkin, Yu. A. Tsidulko, and N. J. Fisch, Phys. Rev. Lett. 85, 4068 (2000).

[12] M. Deutsch, B. Meerson, and J. Golub, Phys. Fluids B 4, 1773 (1991).

[13] R. R. Lindberg, A. E. Charman, J. S. Wurtele, and L. Friedland, Phys. Rev. Lett. 93, 055001 (2004).

[14] O. Polomarov and G. Shvets, Phys. Plasmas 14, 055908
(2007).

[15] L. Friedland, Phys. Rev. Lett. 69, 1749 (1992).

[16] E. A. Williams, B. I. Cohen, L. Divol, M. R. Dorr, J. A. Hittinger, D. E. Hinkel, A. B. Langdon, R. K. Kirkwood, D. H. Froula, and S. H. Glenzer, Phys. Plasmas 11, 231 (2004).

[17] J. Fajans and L. Friedland, Am. J. Phys. 69, 1096 (2001).

[18] L. Friedland, Phys. Rev. E 55, 1929 (1997).

[19] C. J. McKinstrie, A. Simon, and E. A. Williams, Phys. Fluids 27, 2738 (1984).

[20] I. B. Bernstein, Phys. Fluids 18, 320 (1975).

[21] T. P. Coffey, Phys. Fluids 14, 1402 (1971).

[22] A. I. Akhiezer and R. V. Polovin, Sov. Phys. JETP 3, 696 (1956).

[23] M. N. Rosenbluth and C. S. Liu, Phys. Rev. Lett. 29, 701 (1972).

[24] J. Fajans, E. Gilson, and L. Friedland, Phys. Rev. Lett. 82, 4444 (1999).

[25] L. Friedland, Phys. Rev. E 58, 3865 (1998). 